\title{
CENTRAL SECTIONS OF CENTRALLY SYMMETRIC CONVEX BODIES
}

\author{
Dedicated to Professor T. Otsuki on his 70th birthday
}

By SHUKICHI TANNO

\section{§ 0 . Introduction.}

Let $K$ and $K^{\prime}$ be two centrally symmetric convex bodies in the 3-dimensional Euclidean space $E^{3}$ with their centers at the origin $O$. The following problem is still open:

Suppose that for each plane $L$ through $O$ in $E^{3}$

$$
\operatorname{Area}(K \cap L)<\operatorname{Area}\left(K^{\prime} \cap L\right)
$$

holds. Then does the inequality $\operatorname{Vol}(K)<\operatorname{Vol}\left(K^{\prime}\right)$ follow?

This problem has a natural meaning for any dimension $m \geqq 3$ by taking a hyperplane through the origin $O$ of $E^{m}$ as $L$. Let $K$ and $K^{\prime}$ be centrally symmetric convex bodies in $E^{m}$ with their centers at $O$. Then the following are known.

(i) Equality of $(\mathrm{m}-1)$-dimensional volumes $\operatorname{Vol}(K \cap L)=\operatorname{Vol}\left(K^{\prime} \cap L\right)$ for each $L$ implies that $K$ and $K^{\prime}$ are congruent; in particular $\operatorname{Vol}(K)=\operatorname{Vol}\left(K^{\prime}\right)$. This is shown by the generalized Funk's spherical integration theorem, which says that two even functions $f_{1}$ and $f_{2}$ on the $(m-1)$-dimensional unit sphere $S^{m-1}(1)$ are identical, if the integrals of $f_{1}$ and $f_{2}$ on each totally geodesic $(m-2)$-sphere are identical (cf. P. Funk [7], T. Bonnesen and W. Fenchel [2], p. $136-138$, A. L. Besse [1], p. 103-104, p. $124-125$ for $m=3$. Generalization to general $m$ is not difficult.).

(ii) If $K$ is an ellipsoid in $E^{m}$ and $\operatorname{Vol}(K \cap L)<\operatorname{Vol}\left(K^{\prime} \cap L\right)$ holds for each $L$, then $\operatorname{Vol}(K)<\operatorname{Vol}\left(K^{\prime}\right)$ follows (H. Busemann [3]). However, if $K^{\prime}$ is an ellipsoid then the question has not been answered yet.

(iii) By probabilistic arguments, D. G. Larman and C. A. Rogers [9] established the existence of a centrally symmetric convex body $K$ in $E^{m}$, for $m \geqq 12$, such that for each hyperplane $L, \operatorname{Vol}(K \cap L)<\operatorname{Vol}\left(B^{m} \cap L\right)$ holds, nevertheless $\operatorname{Vol}(K)>\operatorname{Vol}\left(B^{m}\right)$, where $B^{m}$ denotes the $m$-dimensional unit ball.

For a general survey on this problem and related subjects see an article

Received May 2, 1987. 
by D. G. Larman [8] in the Proceedings of the International Congress of Mathematicians in Helsinki, 1978.

Now we return to the 3-dimensional case, which seems to be most important at present.

By $B^{3}(R)$ we denote the ball of radius $R$ with center $O$ in $E^{3}$, and by $S^{2}(1)$ we denote the unit sphere in $E^{3}$.

Let $\varepsilon$ be a positive number and $N$ be a natural number. Then $2 N$ points $\pm p_{1}, \pm p_{2}, \cdots, \pm p_{N}$ on $S^{2}(1)$ are called $\varepsilon$-properly distributed on $S^{2}(1)$, if for any two different elements $x, y \in\left\{ \pm p_{1}, \pm p_{2}, \cdots, \pm p_{N}\right\}$ two geodesic $\varepsilon$-disks on $S^{2}(1)$ centered at $x$ and $y$ are disjoint. By $\Theta=\left\{ \pm p_{1}, \pm p_{2}, \cdots, \pm p_{N}\right\}$ we denote an $\varepsilon$-proper distribution of $2 N$ points on $S^{2}(1)$.

By $K(\varepsilon, N, \Theta)$ we denote a centrally symmetric convex body obtained from $B^{3}(1)$ by removing $2 N$ spherical caps of $B^{3}(1)$ of angular radius $\varepsilon$ corresponding to $\Theta . K(\varepsilon, N, \Theta)$ is a natural object as a centrally symmetric convex body which enables us to calculate various quantities and was studied in [9] for seneral dimension $m$.

For each $\varepsilon$-proper distribution $\Theta$ of $2 N$ points on $S^{2}(1)$, if one varies planes $L$ through $O$ in $E^{3}$, then the mean value of $\operatorname{Area}(K(\varepsilon, N, \Theta) \cap L)$ is independent of $\Theta$, and so we denote it by $M(\varepsilon, N)$.

Let $R(\varepsilon, N)$ be a real number determined by

$$
\operatorname{Vol}\left(B^{3}(R(\varepsilon, N))\right)=\operatorname{Vol}(K(\varepsilon, N, \Theta)) \text {. }
$$

Then $R(\varepsilon, N)<1$. If one could define $\Theta$ such that

$$
\operatorname{Area}(K(\varepsilon, N, \Theta) \cap L)<\pi R(\varepsilon, N)^{2}
$$

holds for each $L$, then replacing $R(\varepsilon, N)$ by a slightly smaller $R^{\prime}$, one would get a counter example $K(\varepsilon, N, \Theta)$ :

$$
\begin{gathered}
\operatorname{Area}(K(\varepsilon, N, \Theta) \cap L)<\operatorname{Area}\left(B^{3}\left(R^{\prime}\right) \cap L\right), \\
\operatorname{Vol}(K(\varepsilon, N, \Theta))>\operatorname{Vol}\left(B^{3}\left(R^{\prime}\right)\right) .
\end{gathered}
$$

As Proposition 3.6 we prove the following.

THEOREM A. $M(\varepsilon, N)<\pi R(\varepsilon, N)^{2}$ holds.

This means that the mean value of the left hand side of $(0.1)$ is always smaller than the right hand side. Therefore, at a glance, it seems to be possible to construct counter-examples to the question by distributing $2 N$ points "homogeneously".

The purpose of this paper is to give some evidence that $\pi R(\varepsilon, N)^{2}-M(\varepsilon, N)$ is too small to give $\Theta$ satisfying (0.1).

If $N$ is not so large and $\varepsilon$ is so small, then one may find $L$ which does not meet any removed caps of $K(\varepsilon, N, \Theta)$.

If $N$ is not so large and $\varepsilon$ is so big as possible, then the variation of 
$\operatorname{Area}(K(\varepsilon, N, \Theta) \cap L)$ with respect to $L$ is so big. In $\S 9$ we show two examples related to an octahedron and icosahedron, and one additional example which is not centrally symmetric.

To study the cases where $100 \leqq N<\infty$, we define an ideal homogeneous model $\Theta_{0}^{\prime}$ called the $H$-model of $\varepsilon$-proper distribution of $2 N$ points on $S^{2}(1)$ in $\S 4$. $\Theta_{0}^{\prime}$ is not concrete, but it is an abstract model which is nearly homogeneous and which allows us to calculate necessary quantities for $\varepsilon \rightarrow 0, N \rightarrow \infty$.

THEOREM B. Let $N \geqq 100$. For the $H$-model $\Theta_{0}^{\prime}$, there exists some plane $L$ through $O$ such that

$$
\operatorname{Area}\left(K\left(\varepsilon, N, \Theta_{0}^{\prime}\right) \cap L\right)>\pi R(\varepsilon, N)^{2} \text {. }
$$

\section{$\S 1$. Volumes of spherical caps.}

Let $B^{m}(1)$ be the unit ball with center $O$ in the $m$-dimensional Euclidean space $E^{m}$. For a positive number $\varepsilon$ and a point $p$ in the boundary of $B^{m}(1)$, $\varepsilon$-spherical cap $C^{m}(p, \varepsilon)$ of $B^{m}(1)$ is defined by

$$
C^{m}(p, \varepsilon)=\left\{x \in B^{m}(1) ;(x, p)>\cos \varepsilon\right\},
$$

where $(x, p)$ denotes the inner product of $x$ and $p$, as position vectors. Then the volume of $C^{m}(p, \varepsilon)$ is given by (cf. [9], p. 166).

$$
\operatorname{Vol}\left(C^{m}(p, \varepsilon)\right)=\frac{\pi^{(m-1) / 2}}{\Gamma((m+1) / 2)} \int_{0}^{\varepsilon} \sin ^{m} \theta d \theta
$$

LEMMA 1.1. For $m=2$ and 3 we get

$$
\begin{gathered}
\operatorname{Area}\left(C^{2}(p, \varepsilon)\right)=\varepsilon-\frac{1}{2} \sin 2 \varepsilon, \\
\operatorname{Vol}\left(C^{3}(p, \varepsilon)\right)=\frac{\pi}{3}\left(\cos ^{3} \varepsilon-3 \cos \varepsilon+2\right) .
\end{gathered}
$$

§. Mean value of Area $(K(\varepsilon, N, \Theta) \cap L)$.

In this section we give the expression of the mean value $M(\varepsilon, N)$ of $\operatorname{Area}(K(\varepsilon, N, \Theta) \cap L)$ for an $\varepsilon$-proper distribution $\Theta$ of $2 N$ points on $S^{2}(1)$.

Define a point $A$ in $E^{3}$ by $A=(0,0,1)$, where coordinates of a point or components of a vector are ones with respect to the standard basis of $E^{3}$. Let $K_{0}(\varepsilon)$ denote the unit ball removed one spherical cap $C^{3}(A, \varepsilon) ; K_{0}(\varepsilon)=B^{3}(1)-$ $C^{3}(A, \varepsilon)$. Let $g$ be a great circle on the unit sphere $S^{2}(1)$ in $E^{3}$. Suppose that $g$ meets the geodesic circle on $S^{2}(1)$ of radius $\varepsilon$ centered at $A$ at two points $V$ and $Z$. Let $M$ be the middle point of the (shorter) geodesic segments $V Z$ of g. The length of the geodesic segment $M V$ is denoted by $\varepsilon^{\sim}$ and the distance on $S^{2}(1)$ between $A$ and $M$ is denoted by $t$. Then we get 
The set of all planes through $O$ is identified with a 2-dimensional real projective space $R P^{2}$ by considering to each plane $L$ its normal line through $O$. We identify $R P^{2}$ with $S_{*}^{2}(1)$, which denotes the closed upper hemisphere removed one half of the equator. $R P^{2}$ is also identified with the set of all great circles on $S^{2}(1)$ by identifying $L$ with $L \cap S^{2}(1)=g$. For $x \in S_{*}^{2}(1), g(x)$ or $L(x)$ means the great circle on $S^{2}(1)$ or plane through $O$ corresponding to $x$ with respect to the above identification.

By $P(\varepsilon)$ we denote the mean value of $\pi$-Area $\left(K_{0}(\varepsilon) \cap L\right)$ with respect to $\{L\}=R P^{2}$. Then, the mean value $M(\varepsilon, N)$ of $\operatorname{Area}(K(\varepsilon, N, \Theta) \cap L)$ with respect to $\{L\}$ is given by $\pi-2 N \cdot P(\varepsilon)$.

LEMMA 2.1. Let $\varepsilon^{\sim}=\varepsilon^{\sim}(\varepsilon, t)$ be a function defined by (2.1). Then $P(\varepsilon)$ is given by

$$
P(\varepsilon)=\int_{0}^{\varepsilon}\left(\varepsilon^{\sim}-\frac{1}{2} \sin 2 \varepsilon^{\sim}\right) \cos t d t .
$$

Proof. Let $(s, \theta)$ be a polar coordinate system of $S_{*}^{2}(1)$ centered at $A$. (For a point $x$ in $S_{*}^{2}(1), s=s(x)$ is the distance between $x$ and $A$, and $\theta$ is zero for the geodesic segment $A X$ where $X=(1,0,0)$.) Then the volume element of $S_{*}^{2}(1)$ is given by $\sin s d s d \theta$.

For $x \in S_{*}^{2}(1)$ such that $\pi / 2-\varepsilon \leqq s(x) \leqq \pi / 2, g(x)$ meets the spherical cap $C^{3}(A, \varepsilon)$ of $B^{3}(1)$. For $x \in S_{*}^{2}(1)$ the distance $t=t(x)$ between $A$ and $g(x)$ is equal to $\pi / 2-s(x)$. So, $\varepsilon^{\sim}(\varepsilon, t)$ is determined and

$$
\operatorname{Area}\left(B^{2}(1)\right)-\operatorname{Area}\left(K_{0}(\varepsilon) \cap L(x)\right)=\operatorname{Area}\left(C^{2}\left(M, \varepsilon^{\sim}\right)\right),
$$

where $M$ is the point of $g(x)$ nearest to $A$. By Lemma 1.1 we get

$$
P(\varepsilon)=\frac{1}{2 \pi} \int_{0}^{2 \pi} \int_{0}^{\varepsilon}\left(\varepsilon^{\sim}-\frac{1}{2} \sin 2 \varepsilon^{\sim}\right) \cos t d t d \theta,
$$

where we have used $\operatorname{Vol}\left(R P^{2}\right)=2 \pi$. Thus, proof is completed.

Later we need the following relations among $\varepsilon^{\sim}, \varepsilon$ and $t$. By $\left[\varepsilon^{k}\right]$ we denote the higher order $(\geqq k)$ terms with respect to $\varepsilon, \varepsilon^{\sim}$ and $t$. This is reasonable, because $\varepsilon^{\sim} \leqq \varepsilon$ and $t \leqq \varepsilon$.

LEMMA 2.2. $\boldsymbol{\varepsilon}^{\sim}$ is expanded as follows:

$$
\begin{aligned}
& \varepsilon^{\sim 2}=\left(\varepsilon^{2}-t^{2}\right)\left(1+\frac{1}{3} t^{2}\right)+\left[\varepsilon^{6}\right] . \\
& \varepsilon^{\sim}=\sqrt{\varepsilon^{2}-t^{2}}\left(1+\frac{1}{6} t^{2}\right)+\left[\varepsilon^{5}\right] .
\end{aligned}
$$

Proof. Expanding $\cos \varepsilon, \cos \varepsilon^{\sim}$ and $\cos t$ in each variable and using (2.1) we obtain 


$$
\varepsilon^{\sim 2}=\varepsilon^{2}-t^{2}+\frac{1}{2} t^{2} \varepsilon^{\sim 2}+\frac{1}{12}\left(\varepsilon^{\sim 4}+t^{4}-\varepsilon^{4}\right)+\left[\varepsilon^{6}\right],
$$

and hence we get (2.3). (2.4) follows from (2.3).

\section{§3. $R(\varepsilon, N)$ and $P(\varepsilon)$.}

Let $K(\varepsilon, N, \Theta)$ be a centrally symmetric convex body obtained from $B^{3}(1)$ by removing $2 N$ spherical caps as before. By Lemma 1.1 and

$$
\operatorname{Vol}(K(\varepsilon, N, \Theta))=\frac{4 \pi}{3}-2 N \cdot \operatorname{Vol}\left(C^{3}(A, \varepsilon)\right)
$$

we see that $R(\varepsilon, N)$ satisfying

$$
\operatorname{Vol}(K(\varepsilon, N, \Theta))=\operatorname{Vol}\left(B^{3}(R(\varepsilon, N))\right)
$$

is given by

$$
R(\varepsilon, N)^{3}=1-\frac{N}{2}\left(\cos ^{3} \varepsilon-3 \cos \varepsilon+2\right) .
$$

Then $\operatorname{Area}(K(\varepsilon, N, \Theta) \cap L)<\pi R(\varepsilon, N)^{2}$ is equivalent to

$$
\operatorname{Area}(K(\varepsilon, N, \Theta) \cap L)<\operatorname{Area}\left(B^{3}(R(\varepsilon, N)) \cap L\right) .
$$

We define $A(\varepsilon, N)$ by

$$
A(\varepsilon, N)=\pi\left(1-R(\varepsilon, N)^{2}\right) .
$$

$A(\varepsilon, N)$ is the lower bound of the sum of areas of spherical caps removed in $K(\varepsilon, N, \Theta) \cap L$ for $K(\varepsilon, N, \Theta)$ to satisfy (3.1) and (3.3).

For some pairs $(\varepsilon, N)$ we calculate values of $P(\varepsilon)$ and $A(\varepsilon, N)$ showing the inequality $2 N \cdot P(\varepsilon)>A(\varepsilon, N)$. The difference $2 N \cdot P(\varepsilon)-A(\varepsilon, N)$ may be helpful to understand the situation.

Table 3.1. For pairs $(\varepsilon, N)$ such that $N \varepsilon^{2} \doteqdot 1$ :

\begin{tabular}{lcccc}
\multicolumn{1}{c}{$\boldsymbol{\varepsilon}$} & $N$ & $2 N \cdot P(\varepsilon)$ & $A(\varepsilon, N)$ & $2 N \cdot P(\varepsilon)-A(\varepsilon, N)$ \\
0.1 & 100 & $7.8409 \cdots 10^{-3}$ & $7.8327 \cdots 10^{-3}$ & $8.2 \cdot 10^{-6}$ \\
0.075 & 177 & $4.3944 \cdots 10^{-3}$ & $4.3918 \cdots 10^{-3}$ & $2.6 \cdot 10^{-6}$ \\
0.05 & 400 & $1.9626 \cdots 10^{-3}$ & $1.9621 \cdots 10^{-3}$ & $5.1 \cdot 10^{-7}$ \\
0.025 & 1600 & $4.9082 \cdots 10^{-4}$ & $4.9079 \cdots 10^{-4}$ & $3.2 \cdot 10^{-8}$
\end{tabular}


Table 3.2. For pairs $(\varepsilon, N)$ such that $\varepsilon=0.05$ :

$\begin{array}{lccc}N & 2 N \cdot P(\varepsilon) & A(\varepsilon, N) & 2 N \cdot P(\varepsilon)-A(\varepsilon, N) \\ 100 & 4.9066 \cdots 10^{-4} & 4.9048 \cdots 10^{-4} & 1.9 \cdot 10^{-7} \\ 200 & 9.8133 \cdots 10^{-4} & 9.8100 \cdots 10^{-4} & 3.3 \cdot 10^{-7} \\ 300 & 1.4720 \cdots 10^{-3} & 1.4715 \cdots 10^{-3} & 4.4 \cdot 10^{-7} \\ 500 & 2.4533 \cdots 10^{-3} & 2.4528 \cdots 10^{-3} & 5.4 \cdot 10^{-7}\end{array}$

In the table $3.1, N \varepsilon^{2} \doteqdot 1$ corresponds to the fact that the sum of areas of $2 N$ geodesic $\varepsilon$-disks in $S^{2}(1)$ is about one half of the total area of $S^{2}(1)$. In the table 3.2 , we notice that the number $N$ is limitted by (3.8) below.

LeMma 3.3. For $\varepsilon<0.136, P(\varepsilon)$ is estimated by

$$
\frac{\pi}{8} \varepsilon^{4}-\frac{\pi}{40} \varepsilon^{6}<P(\varepsilon)<\frac{\pi}{8} \varepsilon^{4}-\frac{\pi}{60} \varepsilon^{6} .
$$

Proof. Expanding $\sin 2 \varepsilon^{\sim}$ and using (2.3) and (2.4) we obtain

$$
\begin{aligned}
& \varepsilon^{\sim}-\frac{1}{2} \sin 2 \varepsilon^{\sim}=\frac{2}{3} \varepsilon^{\sim}\left(\varepsilon^{\sim}-\frac{1}{5} \varepsilon^{\sim 4}+\left[\varepsilon^{6}\right]\right) \\
& =\frac{2}{3} \sqrt{\varepsilon^{2}-t^{2}}\left(\varepsilon^{2}-t^{2}-\frac{1}{5} \varepsilon^{4}+\frac{9}{10} \varepsilon^{2} t^{2}-\frac{7}{10} t^{4}\right)+\left[\varepsilon^{7}\right] .
\end{aligned}
$$

Expanding $\cos t$ we get

$$
\begin{aligned}
P(\varepsilon)= & \int_{0}^{\varepsilon}\left(\varepsilon^{2}-\frac{1}{2} \sin 2 \varepsilon^{2}\right) \cos t d t \\
= & \frac{2}{3}\left(\varepsilon^{2}-\frac{1}{5} \varepsilon^{4}\right) \int_{0}^{\varepsilon} \sqrt{\varepsilon^{2}-t^{2}} d t-\frac{2}{3}\left(1-\frac{2}{5} \varepsilon^{2}\right) \int_{0}^{\varepsilon} \sqrt{\varepsilon^{2}-t^{2}} t^{2} d t \\
& -\frac{2}{15} \int_{0}^{\varepsilon} \sqrt{\varepsilon^{2}-t^{2}} t^{4} d t+\left[\varepsilon^{8}\right] .
\end{aligned}
$$

On the other hand, for each even integer $k$, we have

$$
\int_{0}^{\varepsilon} \sqrt{\varepsilon^{2}-t^{2}} t^{k} d t=\frac{\pi(k-1) ! !}{2(k+2) ! !} \varepsilon^{k+2}
$$

and so we get

$$
P(\varepsilon)=\frac{\pi}{8} \varepsilon^{4}-\frac{\pi}{48} \varepsilon^{6}+\left[\varepsilon^{8}\right] .
$$

For $\varepsilon<0.136$ by numerical calculation (by computer) we can verify (3.5). For example, if $\varepsilon=0.136$, then

$$
\pi / 8-\pi \varepsilon^{2} / 40=0.3912 \cdots,
$$




$$
\begin{aligned}
P(\varepsilon) / \varepsilon^{4} & =0.3914 \cdots, \\
\pi / 8-\pi \varepsilon^{2} / 60 & =0.3917 \cdots .
\end{aligned}
$$

The meaning of the value 0.136 is explained later in $\S 5$.

LEMMA 3.4. For $\varepsilon<\pi / 2, N \varepsilon^{2}$ is estimated by

$$
N \varepsilon^{2}<2+\frac{1}{5} \varepsilon^{2}
$$

Proof. The area of a geodesic disk of radius $\varepsilon$ on $S^{2}(1)$ is $2 \pi(1-\cos \varepsilon)$. So the total area of $2 N$ geodesic disks is $4 \pi N(1-\cos \varepsilon)$, which is smaller than the area $4 \pi$ of $S^{2}(1)$. Expanding $\cos \varepsilon$ we get the inequality.

LemMA 3.5. For $\varepsilon<0.136, A(\varepsilon, N)$ is estimated by

$$
\pi N\left(\frac{1}{4} \varepsilon^{4}-\frac{1}{12} \varepsilon^{6}\right)<A(\varepsilon, N)<\pi N\left(\frac{1}{4} \varepsilon^{4}-\frac{1}{20} \varepsilon^{6}\right) .
$$

Proof. Since

$$
\cos ^{3} \varepsilon-3 \cos \varepsilon+2=\frac{3}{4} \varepsilon^{4}-\frac{1}{4} \varepsilon^{6}+\frac{13}{320} \varepsilon^{8}+\left[\varepsilon^{10}\right],
$$

by (3.2) we obtain the expansion of $R(\varepsilon, N)^{3}$ and hence

$$
\begin{aligned}
R(\varepsilon, N)=1- & \frac{N}{8} \varepsilon^{4}+\frac{N}{24} \varepsilon^{6}-\left(\frac{13 N}{1920}+\frac{N^{2}}{64}\right) \varepsilon^{8} \\
& +N\left[\varepsilon^{10}\right]+N^{2}\left[\varepsilon^{10}\right]+\sum_{h=3}^{\infty} N^{h}\left[\varepsilon^{4 h}\right] .
\end{aligned}
$$

Furthermore we get

$$
\begin{aligned}
R(\varepsilon, N)^{2}= & 1-\frac{N}{4} \varepsilon^{4}+\frac{N}{12} \varepsilon^{6}-\left(\frac{13 N}{960}+\frac{N^{2}}{64}\right) \varepsilon^{8} \\
& +N\left[\varepsilon^{10}\right]+N^{2}\left[\varepsilon^{10}\right]+\sum_{h=3}^{\infty} N^{h}\left[\varepsilon^{4 h}\right] .
\end{aligned}
$$

Since $N \varepsilon^{2}$ is bounded, we can put $N^{h}\left[\varepsilon^{4 h}\right]=N\left[\varepsilon^{2 h+2}\right]$ and so

$$
R(\varepsilon, N)^{2}=1-\frac{N}{4} \varepsilon^{4}+\frac{N}{12} \varepsilon^{6}-\frac{N^{2}}{64} \varepsilon^{8}+N\left[\varepsilon^{8}\right] .
$$

Therefore

$$
A(\varepsilon, N)=\pi N\left(\frac{1}{4} \varepsilon^{4}-\frac{1}{12} \varepsilon^{6}+\frac{N}{64} \varepsilon^{8}+\left[\varepsilon^{8}\right]\right) .
$$

We use (3.8) to obtain the upper estimate of $A(\varepsilon, N)$ and we replace $N \varepsilon^{8} / 64$ in (3.10) by $\varepsilon^{6} / 32$. Then, (3.9) is verified by numerical calculation. For each 
value of $\varepsilon$ the range of $N$ is limited by (3.8).

For example, if $\varepsilon=0.136$ (in this case $1 \leqq N \leqq 108$ ) and if $N=100$, then

$$
\begin{array}{r}
\pi N\left(1 / 4-\varepsilon^{2} / 12\right)=78.05 \cdots, \\
A(\varepsilon, N) / \varepsilon^{4}=78.22 \cdots, \\
\pi N\left(1 / 4-\varepsilon^{2} / 20\right)=78.24 \cdots .
\end{array}
$$

Now we prove the following.

Proposition 3.6. $2 N \cdot P(\varepsilon)>A(\varepsilon, N)$ holds for each pair $(\varepsilon, N)$ such that $2 N$ points can be $\varepsilon$-properly distributed on $S^{2}(1)$.

Proof. For $\varepsilon<0.136$ we see that $2 N \cdot P(\varepsilon)>A(\varepsilon, N)$ by the first inequality of (3.5) and the second inequality of (3.9).

For $\varepsilon \geqq 0.136$ we can verify $2 N \cdot P(\varepsilon)>A(\varepsilon, N)$ by numerical calculation. For each value of $\varepsilon$, the range of $N$ is limitted by (3.8). If $\varepsilon$ gets larger, then the maximum of $N$ gets smaller.

\section{$\S 4$. $H$-model.}

From now on we show some evidence that

$$
\pi R(\varepsilon, N)^{2}-M(\varepsilon, N)=2 N \cdot P(\varepsilon)-A(\varepsilon, N)
$$

is too small to construct concrete examples $K(\varepsilon, N, \Theta)$ satisfying (3.1) and (3.3).

We define $\Theta_{0}^{\prime}=\left\{ \pm q_{1}, \pm q_{2}, \cdots, \pm q_{N}\right\}$ somewhat abstractly. First we define $q_{1}, q_{2}$ and $q_{3}$ in the following setting.

$\langle 4-1\rangle$ Setting.

(i) $\left\{q_{1}, q_{2}, q_{3}\right\}$ makes an equilateral (geodesic) triangle on $S^{2}(1)$.

(ii) The center of the triangle $q_{1} q_{2} q_{3}$ is $A=(0,0,1)$.

(iii) For each $i(i=1,2,3), q_{\imath}$ represents a hexagon $H_{\imath}$ on $S^{2}(1)$ and $q_{\imath}$ is the center of $H_{2}$.

(iv) The area of $H_{\imath}$ is equal to $4 \pi / 2 N$.

(v) $H_{1}, H_{2}$ and $H_{3}$ are placed naturally so that two edges of each $H_{2}$ coincide with respective one edge of the other hexagons.

$\langle 4-2\rangle$ Definition of hexagon $H\left(=H_{1}\right)=A B C D E F$.

(i) $A, D$ and the center $Q\left(=q_{1}\right)$ of $H$ are in the $\left(x^{2}, x^{3}\right)$-plane.

(ii) The lengths of the geodesic segments $A B, B Q, Q C, C D, D E, E Q, Q F$, $F A$ are all equal to $\alpha$.

(iii) $\angle B A Q=\angle F A Q=\angle C D Q=\angle E D Q=\pi / 3$.

Coordinate expressions of these points are as follows:

$$
\begin{aligned}
& A=(0,0,1), \\
& B=((\sqrt{3} / 2) \sin \alpha,(1 / 2) \sin \alpha, \cos \alpha),
\end{aligned}
$$




$$
\begin{gathered}
C=\left(c^{1}, c^{2}, c^{3}\right) / 2\left(3 \cos ^{2} \alpha+1\right), \\
c^{1}=\sqrt{3}\left(3 \cos ^{2} \alpha+1\right) \sin \alpha, \\
c^{2}=\left(13 \cos ^{2} \alpha-1\right) \sin \alpha, \\
c^{3}=14 \cos ^{3} \alpha-6 \cos \alpha, \\
D=\left(0, d^{2}, d^{3}\right) /\left(3 \cos ^{2} \alpha+1\right)^{2}, \\
d^{2}=8\left(5 \cos ^{2} \alpha-1\right) \cos \alpha \sin \alpha, \\
d^{3}=41 \cos ^{4} \alpha-26 \cos ^{2} \alpha+1, \\
Q=\left(0,4 \cos \alpha \sin \alpha, 5 \cos ^{2} \alpha-1\right) /\left(3 \cos ^{2} \alpha+1\right) .
\end{gathered}
$$

$\langle 4-3\rangle$ Area of $H$.

We denote the lengths of geodesic segments $A Q$ and $B C$ by $\lambda$ and $\mu$, respectively. Then we get

$$
\begin{aligned}
& \cos \lambda=(A, Q)=\left(5 \cos ^{2} \alpha-1\right) /\left(3 \cos ^{2} \alpha+1\right), \\
& \cos \mu=(B, C)=\left(\cos ^{2} \alpha+1\right) / 2 .
\end{aligned}
$$

With respect to the triangles $A B Q$ and $B C Q$ we get the classical relations:

$$
\begin{aligned}
& \sin \alpha \sin \angle A B Q=\sin \lambda \sin (\pi / 3) \\
& \sin \alpha \sin (\pi / 3)=\sin \mu \sin \angle Q B C
\end{aligned}
$$

and we can calculate $\angle A B Q$ and $\angle Q B C$. Further, we obtain

$$
\operatorname{Area}(H)=4(<A B Q+<Q B C)-8 \pi / 3 \text {. }
$$

For a given value of $\alpha$, we can calculate $\angle A B Q$ and $\angle Q B C$ by (4.1) and (4.2). Next by (4.3) we obtain the area $\operatorname{Area}(H)$ corresponding to $\alpha$. Conversely, for a given natural number $N$ we can find the (approximated) value of $\alpha$ so that $\operatorname{Area}(H)=4 \pi / 2 N$.

Example. (i) For $N=100, \alpha=0.1551 \cdots$.

(ii) For $N=400$, we get the following values:

$$
\begin{array}{ll}
\operatorname{Area}(H)=2 \pi / N=0.0157 \cdots & \alpha=0.0777 \cdots \\
\lambda=0.0778 \cdots & \mu=0.0776 \cdots \\
<A B Q=1.0498 \cdots & <Q B C=1.0485 \cdots
\end{array}
$$

LemMA 4.1. Area $(H)=2 \pi / N$ is expanded as follows:

$$
\frac{2 \pi}{N}=\frac{3 \sqrt{3}}{2} \alpha^{2}+\frac{5 \sqrt{3}}{16} \alpha^{4}+\left[\alpha^{6}\right] \text {. }
$$


Proof. Expanding $\cos \lambda$ and $\cos \mu$ with respect to $\alpha$, we get

$$
\begin{aligned}
& \cos \lambda=1-\frac{1}{2} \alpha^{2}-\frac{5}{24} \alpha^{4}-\frac{77}{1440} \alpha^{6}+\left[\alpha^{8}\right], \\
& \cos \mu=1-\frac{1}{2} \alpha^{2}+\frac{1}{6} \alpha^{4}-\frac{1}{45} \alpha^{6}+\left[\alpha^{8}\right],
\end{aligned}
$$

and using relations (4.1) and (4.2) we obtain

$$
\begin{aligned}
& \sin \angle A B Q=\frac{\sqrt{3}}{2}\left(1+\frac{1}{4} \alpha^{2}-\frac{1}{48} \alpha^{4}+\left[\alpha^{6}\right]\right), \\
& \sin \angle Q B C=\frac{\sqrt{3}}{2}\left(1+\frac{1}{8} \alpha^{2}-\frac{7}{384} \alpha^{4}+\left[\alpha^{6}\right]\right) .
\end{aligned}
$$

$\cos \angle A B Q$ and $\cos \angle Q B C$ are obtained from these. We put $4 Z=\operatorname{Area}(H)$. Expanding $\sin (<A B Q+<Q B C)=\sin (Z+2 \pi / 3)$, we obtain

$$
\frac{\sqrt{3}}{4}\left(\frac{3}{4} \alpha^{2}+\frac{111}{192} \alpha^{4}+\left[\alpha^{6}\right]\right)=\frac{1}{2} Z+\frac{\sqrt{3}}{4} Z^{2}+\left[Z^{3}\right],
$$

from which we obtain the relation in Lemma 4.1.

By Lemma 4.1 we get

$$
\alpha^{2} N=\frac{4 \sqrt{3}}{9} \pi-\frac{5 \sqrt{3}}{54} \pi \alpha^{2}+\left[\alpha^{4}\right],
$$

and by numerical calculation we can verify $\alpha^{2} N>4 \sqrt{3} \pi / 9-\pi \alpha^{2} / 5$ for $\alpha<0.156$. Then $\alpha^{2} N>2.4$ for $\alpha<0.156$, and hence we get the following.

LEMMA 4.2. For $\alpha<0.156, \alpha N$ is estimated by

$$
\alpha N>\frac{12}{5 \alpha} \text {. }
$$

$\langle 4-4\rangle$ Mean values.

Let $\Omega$ be the domain in $S^{2}(1)$ defined by three hexagons $H_{1}, H_{2}$, and $H_{3}$. Since $q_{1}(=Q), q_{2}$ and $q_{3}$ are defined as centers of three hexagons, to define $\Theta_{0}^{\prime}$ as a standard model we suppose that $2 N-6$ points $\left\{ \pm q_{4}, \cdots, \pm q_{N}\right\}$ are distributed in $S^{2}(1)-\Omega \cup(-\Omega)$ (abstractly and) nearly homogeneously.

Let $\left\{L_{A}\right\}$ be the set of all planes which contain the line $A O$. Planes $L_{A}$ are parametrized by angles $\theta$ from the first axis; $0 \leqq \theta<\pi$. We want to calculate the rotational mean value at $A$, that is, the mean value $M_{0}^{\prime}(\varepsilon, N ; A)$ of $\operatorname{Area}\left(K\left(\varepsilon, N, \Theta_{0}^{\prime}\right) \cap L_{A}\right)$ with respect to planes $\left\{L_{A}\right\}$.

Let $\Theta_{0}$ be an abstract $\varepsilon$-proper distribution of $2 N$ points on $S^{2}(1)$, which is nearly homogeneously distributed. Then the mean value $M_{0}(\varepsilon, N ; A)$ of $\operatorname{Area}\left(K\left(\varepsilon, N, \Theta_{0}\right) \cap L_{A}\right)$ with respect to $\left\{L_{A}\right\}$ is equal to $M(\varepsilon, N)=\pi-2 N \cdot P(\varepsilon)$. Here we divide $2 N \cdot P(\varepsilon)$ into two factors: 


$$
2 N \cdot P(\varepsilon)=2 P(\varepsilon, N, \Omega)+P\left(\varepsilon, N, S^{2}(1)-\Omega \cup(-\Omega)\right),
$$

where $P(\varepsilon, N, \Omega)$ is defined as follows: Let $\theta \in[\pi / 6, \pi / 2]$ and let $\left\{\rho_{\theta}(s)\right\}$ be the geodesic emanating from $A$ such that the angle between $d \rho_{\theta}(0) / d s$ and the $x^{1}$-axis is $\theta$. By $l(\theta)$ we denote the length of the geodesic segment $\left\{\rho_{\theta}(s)\right\} \cap \Omega$. Then the effect of the mean value of sum of areas of removed caps restricted to $\left\{\rho_{\theta}(s)\right\} \cap \Omega$ is $2 N \cdot P(\varepsilon) \cdot l(\theta) / 2 \pi$, and

$$
P(\varepsilon, N, \Omega)=\frac{6}{\pi} \cdot 2 N \cdot P(\varepsilon) \cdot \frac{1}{2 \pi} \int_{\pi / 6}^{\pi / 2} l(\theta) d \theta .
$$

We denote the mean value of $\pi$-Area $\left(K\left(\varepsilon, N, \Theta^{\prime}\right) \cap L_{A}\right)$ with respect to $\left\{L_{A}\right\}$ by $2 P\left(q_{1} q_{2} q_{3}\right)$, where $\Theta^{\prime}=\left\{ \pm q_{1}, \pm q_{2}, \pm q_{3}\right\}$. To define $\Theta_{0}^{\prime}$ we replace $P(\varepsilon, N, \Omega)$ by $P\left(q_{1} q_{2} q_{3}\right)$.

Definition. $H$-model $\Theta_{0}^{\prime}$ of $\varepsilon$-proper distribution of $2 N$ points on $S^{2}(1)$ is $\left\{ \pm q_{1}, \pm q_{2}, \pm q_{3}, \cdots, \pm q_{N}\right\}$ such that $M_{0}^{\prime}(\varepsilon, N ; A)$ is calculated by

$$
\pi-M_{0}^{\prime}(\varepsilon, N ; A)=2 N \cdot P(\varepsilon)-2 P(\varepsilon, N, \Omega)+2 P\left(q_{1} q_{2} q_{3}\right) .
$$

Here we notice that the condition (i) of Setting $\langle 4-1\rangle$ is related to the case where $N$ is not small. Since we are studying the case where $N \geqq 100$, this may be natural.

\section{$\S 5$. The range of $\varepsilon$ with respect to $\alpha$.}

For a given value of $N, \alpha$ and $H$ are determined. Let $M^{\prime}$ be the middle point of the geodesic segment $A B$. Let $\nu$ be the distance between $M^{\prime}$ and $Q$. Then the range of $\varepsilon$ is estimated by $0<\varepsilon<\nu$. The coordinates of $M^{\prime}$ are given by

$$
(\sqrt{3} \sin \alpha, \sin \alpha, 2(\cos \alpha+1)) /[8(\cos \alpha+1)]^{1 / 2} \text {. }
$$

Therefore

$$
\begin{aligned}
\cos \nu & =\left(M^{\prime}, Q\right) \\
& =\left(3 \cos ^{3} \alpha+5 \cos ^{2} \alpha+\cos \alpha-1\right) /\left(3 \cos ^{2} \alpha+1\right)(2 \cos \alpha+2)^{1 / 2} \\
& =1-\frac{3}{8} \alpha^{2}-\frac{17}{128} \alpha^{4}+\left[\alpha^{6}\right] .
\end{aligned}
$$

Furthermore we obtain

$$
\nu=\frac{\sqrt{3}}{2} \alpha+\frac{5 \sqrt{3}}{48} \alpha^{3}+\left[\alpha^{5}\right]
$$

Consequently

$$
\left(\frac{\nu}{\sin \alpha}\right)^{2}=\frac{3}{4}+\frac{9}{16} \alpha^{2}+\left[\alpha^{4}\right]
$$


and hence

$$
\left[1-\left(\frac{\nu}{\sin \alpha}\right)^{2}\right]^{-1 / 2}=2+\frac{9}{4} \alpha^{2}+\left[\alpha^{4}\right]
$$

By numerical calculation we get the following.

LEMMA 5.1. For $\alpha<0.156$

$$
\begin{gathered}
\left(\frac{\varepsilon}{\sin \alpha}\right)^{2}<\frac{3}{4}+\frac{3}{5} \alpha^{2}, \\
{\left[1-\left(\frac{\varepsilon}{\sin \alpha}\right)^{2}\right]^{-1 / 2}<2+3 \alpha^{2} .}
\end{gathered}
$$

For $N=100$, we get $\alpha=0.1551 \cdots$ and $\nu=0.1350 \cdots$. Since we are studying the case where $N \geqq 100$, the ranges of $\alpha$ and $\varepsilon$ may be set as follows:

$$
0<\alpha<0.156, \quad 0<\varepsilon<0.136
$$

§ 6. $P(\varepsilon, N, \Omega)$.

By $S=A B C D$ we denote the quadrangle on $S^{2}(1)$ defined by $A, B, C$ and $D$. Let $S^{*}=A^{*} B^{*} C^{*} D^{*}$ be the quadrangle on the tangent space $T_{A} S^{2}(1)$ to $S^{2}(1)$ at $A$ satisfying the following conditions.

(i) $\left|A^{*} B^{*}\right|=\left|B^{*} C^{*}\right|=\left|C^{*} D^{*}\right|=\alpha,\left|A^{*} D^{*}\right|=2 \alpha$.

(ii) By the exponential map $\varphi$ at $A, \varphi\left(A^{*}\right)=A, \varphi\left(B^{*}\right)=B$, and $\varphi\left(A^{*} D^{*}\right)$ is contained in the geodesic segment $A D$.

Then $<D^{*} A^{*} B^{*}=\pi / 3$ follows.

LEMMA 6.1. $\varphi^{-1}(S)$ contains $S^{*}$.

Proof. We define $C_{*}$ and $D_{*}$ by $C_{*}=\varphi^{-1}(C)$ and $D_{*}=\varphi^{-1}(D) . \quad<C_{*} A^{*} D^{*}$ is calculated by the coordinates of $C$;

$$
\cos <C_{*} A^{*} D^{*}=\left(13 \cos ^{2} \alpha-1\right) / 2\left(49 \cos ^{4} \alpha-2 \cos ^{2} \alpha+1\right)^{1 / 2} .
$$

Then $\cos ^{2}<C_{*} A^{*} D^{*}-3 / 4<0$ is equivalent to

$$
\left(11 \cos ^{2} \alpha+1\right)\left(\cos ^{2} \alpha-1\right)<0,
$$

and so we see that $<C_{*} A^{*} D^{*}><C^{*} A^{*} D^{*}=\pi / 6$.

Next we show that the orthogonal projection of $C_{*}$ to the line $A^{*} C^{*}$ lies in the extension of $A^{*} C^{*}$. That is,

$$
\left|A^{*} C_{*}\right| \cos \left(<C_{*} A^{*} D^{*}-\pi / 6\right)>\sqrt{3} \alpha .
$$

By the expression of $\cos <C_{*} A^{*} D^{*}$ we get

$$
\cos \left(<C_{*} A^{*} D^{*}-\pi / 6\right)=4 \sqrt{3} \cos ^{2} \alpha /\left(49 \cos ^{4} \alpha-2 \cos ^{2} \alpha+1\right)^{1 / 2} .
$$


Then (6.1) is equivalent to

$$
\left|A^{*} C_{*}\right|>\left(49 \cos ^{4} \alpha-2 \cos ^{2} \alpha+1\right)^{1 / 2} \alpha / 4 \cos ^{2} \alpha .
$$

Since $\left|A^{*} C_{*}\right|=|A C|$, and $\cos |A C|$ is known by the coordinates of $C,(6.2)$ is equivalent to

$$
\left(7 \cos ^{3} \alpha-3 \cos \alpha\right) /\left(3 \cos ^{2} \alpha+1\right)<\cos \beta,
$$

where

$$
\beta=\left(49 \cos ^{4} \alpha-2 \cos ^{2} \alpha+1\right)^{1 / 2} \alpha / 4 \cos ^{2} \alpha .
$$

We expand the both sides of (6.3) and get

$$
\begin{gathered}
\left(7 \cos ^{3} \alpha-3 \cos ^{2} \alpha\right) /\left(3 \cos ^{2} \alpha+1\right)=1-\frac{3}{2} \alpha^{2}+\frac{1}{8} \alpha^{4}+\left[\alpha^{6}\right] \\
<1-\frac{3}{2} \alpha^{2}+\frac{1}{4} \alpha^{4} \quad \text { for } \quad \alpha<0.156 \\
\cos \beta=1-\frac{3}{2} \alpha^{2}+\frac{3}{8} \alpha^{4}+\left[\alpha^{6}\right] \\
>1-\frac{3}{2} \alpha^{2}+\frac{1}{4} \alpha^{4} \quad \text { for } \quad \alpha<0.156 .
\end{gathered}
$$

Therefore we get (6.1). Since $\varphi^{-1}(B C)$ and $\varphi^{-1}(C D)$ are convex in $T_{A} S^{2}(1)$, we see that $\varphi^{-1}(S)$ contains $S^{*}$.

(q. e.d.)

By Lemma 6.1 we obtain

$$
\int_{\pi / 6}^{\pi / 2} l(\theta) d \theta>\int_{\pi / 6}^{\pi / 3} \frac{\sqrt{3} \alpha}{2 \cos \theta} d \theta+\int_{\pi / 3}^{\pi / 2} \frac{\sqrt{3} \alpha}{\cos (\theta-\pi / 3)} d \theta
$$

Since

$$
\int \frac{1}{\cos \theta} d \theta=\log \tan \left(\frac{\theta}{2}+\frac{\pi}{4}\right)
$$

we obtain the following.

$$
P(\varepsilon, N, \Omega)>\frac{3 \sqrt{3}}{\pi^{2}} N \cdot P(\varepsilon) \cdot \alpha\left(\log \tan \frac{5 \pi}{12}+\log \tan \frac{\pi}{3}\right) .
$$

Therefore we get

LEMMA 6.2. $P(\varepsilon, N, \Omega)$ is estimated by

(6.4) $2 P(\varepsilon, N, \Omega)>\frac{3 \sqrt{3}}{\pi^{2}} \log (3+2 \sqrt{3}) \cdot 2 N \cdot P(\varepsilon) \cdot \alpha>\frac{98 \alpha}{100} \cdot 2 N \cdot P(\varepsilon)$. 
§ 7. Mean value $U(b, \varepsilon)$.

Let $X=(1,0,0)$ and $Y=(0,1,0)$. Let $T=(\sin b, 0, \cos b)$ where $0<b \leqq \pi / 2$. Let $L_{A}$ be a plane in $\left\{L_{A}\right\}$ and let $g=g\left(L_{A}\right)=g(\theta)$ be the corresponding great circle on $S^{2}(1), 0 \leqq \theta<\pi / 2$. The point of intersection of $g$ and the equator is $(\cos \theta, \sin \theta, 0)$.

The distance $w=w(b, \theta)$ between $T$ and $g$ is given by

$$
\sin w=\sin b \sin \theta .
$$

Let $\varepsilon<b$ and let $\theta_{0}$ be the value of parameter of $L_{A}$ for which $g$ is tangent to the geodesic circle $C[T, \varepsilon]$ of radius $\varepsilon$ centered at $T$ in $S^{2}(1)$. By putting $w=\varepsilon$ in (7.1) we see that $\theta_{0}$ is determined by

$$
\sin \theta_{0}=\frac{\sin \varepsilon}{\sin b} .
$$

For $\theta \in\left[0, \theta_{0}\right]$, we denote the points of intersection of $g(\theta)$ and $C[T, \varepsilon]$ by $V$ and $Z$. The half of the distance between $V$ and $Z$ is denoted by $\varepsilon^{*}=\varepsilon^{*}(\varepsilon, b, \theta)$. Then

$$
\cos \varepsilon=\cos \varepsilon^{*} \cos w .
$$

With respect to only one spherical cap $C^{3}(T, \varepsilon)$, the mean value $U(b, \varepsilon)$ of areas of removed caps with respect to $\left\{L_{A}\right\}$ is calculated by

$$
U(b, \varepsilon)=\frac{2}{\pi} \int_{0}^{\theta_{0}}\left(\varepsilon^{*}-\frac{1}{2} \sin 2 \varepsilon^{*}\right) d \theta .
$$

If one changes the variables, then (7.4) is rewritten as

$$
U(b, \varepsilon)=\frac{2}{\pi} \int_{0}^{\varepsilon}\left(\varepsilon^{*}-\frac{1}{2} \sin 2 \varepsilon^{*}\right) \frac{\cos w}{\sqrt{\sin ^{2} b-\sin ^{2} w}} d w .
$$

Next we obtain an estimate of $U(\alpha, \varepsilon)$.

LEMMA 7.1.

$$
\begin{aligned}
& \int_{0}^{\varepsilon} \frac{\sqrt{\varepsilon^{2}-w^{2}}}{\sqrt{\sin ^{2} b-w^{2}}} w^{k} d w \\
= & \frac{1}{\sin b}\left[\sum_{l=0}^{\infty} \frac{(2 l-1) ! !}{l ! 2^{l}}\left(\frac{\varepsilon}{\sin b}\right)^{2 l} \frac{\pi(k+2 l-1) ! !}{2(k+2 l+2) ! !}\right] \varepsilon^{k+2} .
\end{aligned}
$$

Proof. By

$$
\frac{1}{\sqrt{1-x}}=\sum_{l=0}^{\infty} \frac{(2 l-1) ! !}{l ! 2^{l}} x^{l}
$$

and (3.6) we obtain 


$$
\begin{aligned}
\int_{0}^{\varepsilon} \frac{\sqrt{\varepsilon^{2}-w^{2}}}{\sqrt{\sin ^{2} b-w^{2}}} w^{k} d w & =\int_{0}^{\varepsilon} \frac{\sqrt{\varepsilon^{2}-w^{2}}}{\sin b}\left[\sum_{l=0}^{\infty} \frac{(2 l-1) ! !}{l ! 2^{l}}\left(\frac{w}{\sin b}\right)^{2 l}\right] w^{k} d w \\
& =\sum_{l=0}^{\infty} \frac{(2 l-1) ! !}{l ! 2^{l} \sin ^{2 l+1} b} \int_{0}^{\varepsilon} \sqrt{\varepsilon^{2}-w^{2}} w^{k+2 l} d w \\
& =\sum_{l=0}^{\infty} \frac{(2 l-1) ! !}{l ! 2^{l} \sin ^{2 l+1} b} \cdot \frac{\pi(k+2 l-1) ! !}{2(k+2 l+2) ! !} \varepsilon^{k+2 l+2},
\end{aligned}
$$

from which we obtain (7.6).

Since $\varepsilon^{*}, \varepsilon$ and $w$ satisfy the relations satisfied by $\varepsilon^{\sim}, \varepsilon$ and $t$, we have the corresponding equalities as in Lemma 2.2. So as in the proof of Lemma 3.3 we obtain

(7.7) $\left(\varepsilon^{*}-\frac{1}{2} \sin 2 \varepsilon^{*}\right) \cos w=\frac{2}{3} \sqrt{\varepsilon^{2}-w^{2}}\left[\varepsilon^{2}-\frac{1}{5} \varepsilon^{4}-\left(1-\frac{2}{5} \varepsilon^{2}\right) w^{2}-\frac{1}{5} w^{4}\right]+\left[\varepsilon^{7}\right]$.

By (7.5) with $b=\alpha$ we get

$$
U(\alpha, \varepsilon)<\frac{2}{\pi} \int_{0}^{\varepsilon}\left(\varepsilon^{*}-\frac{1}{2} \sin 2 \varepsilon^{*}\right) \frac{\cos w}{\sqrt{\sin ^{2} \alpha-w^{2}}} d w .
$$

Applying (7.6) and (7.7) to the last inequality we obtain

$$
\begin{aligned}
& U(\alpha, \varepsilon)<\frac{2}{3}\left(1-\frac{1}{5} \varepsilon^{2}\right) \frac{\varepsilon^{4}}{\sin \alpha}\left[\frac{1}{2}+\frac{1}{2}\left(\frac{\varepsilon}{\sin \alpha}\right)^{2} \frac{1}{4 ! !}+\frac{3}{2 \cdot 4}\left(\frac{\varepsilon}{\sin \alpha}\right)^{4} \frac{3 ! !}{6 ! !}+\cdots\right] \\
& -\frac{2}{3}\left(1-\frac{2}{5} \varepsilon^{2}\right) \frac{\varepsilon^{4}}{\sin \alpha}\left[\frac{1}{4 ! !}+\frac{1}{2}\left(\frac{\varepsilon}{\sin \alpha}\right)^{2} \frac{3 ! !}{6 ! !}+\frac{3}{2 \cdot 4}\left(\frac{\varepsilon}{\sin \alpha}\right)^{4} \frac{5 ! !}{8 ! !}+\cdots\right] \\
& -\frac{2 \varepsilon^{6}}{15 \sin \alpha}\left[\frac{3 ! !}{6 ! !}+\frac{1}{2}\left(\frac{\varepsilon}{\sin \alpha}\right)^{2} \frac{5 ! !}{8 ! !}+\frac{3}{2 \cdot 4}\left(\frac{\varepsilon}{\sin \alpha}\right)^{4} \frac{7 ! !}{10 ! !}+\cdots\right] \\
& \quad+\arcsin \left(\frac{\varepsilon}{\sin \alpha}\right)\left[\varepsilon^{7}\right] \\
& =\frac{2 \varepsilon^{4}}{3 \sin \alpha}\left[\frac{3}{8}+\frac{1}{2}\left(\frac{\varepsilon}{\sin \alpha}\right)^{2} \cdot \frac{1}{16}+\frac{3}{8}\left(\frac{\varepsilon}{\sin \alpha}\right)^{4} \cdot \frac{3}{128}+\cdots\right] \\
& \quad-\frac{2 \varepsilon^{6}}{15 \sin \alpha}\left[\frac{5}{16}+\frac{1}{2}\left(\frac{\varepsilon}{\sin \alpha}\right)^{2} \cdot \frac{5}{128}+\frac{3}{8}\left(\frac{\varepsilon}{\sin \alpha}\right)^{4} \cdot \frac{3}{256}+\cdots\right] \\
& \quad+\arcsin \left(\frac{\varepsilon}{\sin \alpha}\right)\left[\varepsilon^{7}\right] .
\end{aligned}
$$

Since

$$
\left\{\frac{(2 l-1) ! !}{(2 l+2) ! !}-\frac{(2 l+1) ! !}{(2 l+4) ! !}\right\}=\left\{\frac{3}{8}, \frac{1}{16}, \frac{3}{128}, \cdots\right\}
$$

is decreasing with respect to $l$, and 


$$
\left\{\frac{15(2 l-1) ! !}{(2 l+6) ! !}\right\}=\left\{\frac{5}{16}, \frac{5}{128}, \frac{3}{256}, \cdots\right\}
$$

is composed of positive numbers, we obtain

$$
\begin{aligned}
U(\alpha, \varepsilon)< & \frac{2 \varepsilon^{4}}{3 \sin \alpha}\left[\left(\frac{3}{8}-\frac{3}{128}\right)+\frac{1}{2}\left(\frac{\varepsilon}{\sin \alpha}\right)^{2}\left(\frac{1}{16}-\frac{3}{128}\right)\right. \\
& \left.+\frac{3}{128} \sum_{l=0}^{\infty} \frac{(2 l-1) ! !}{l ! 2^{l}}\left(\frac{\varepsilon}{\sin \alpha}\right)^{2 l}\right]-\frac{2 \varepsilon^{6}}{15 \sin \alpha} \cdot \frac{5}{16}+\left[\varepsilon^{8}\right] \\
= & \frac{2 \varepsilon^{4}}{3 \sin \alpha}\left[\frac{45}{128}+\frac{5}{256}\left(\frac{\varepsilon}{\sin \alpha}\right)^{2}+\frac{3}{128 \sqrt{1-(\varepsilon / \sin \alpha)^{2}}}\right] \\
& -\frac{\varepsilon^{6}}{24 \sin \alpha}+\left[\varepsilon^{8}\right] .
\end{aligned}
$$

For $\alpha<0.156$ by Lemma 5.1 we obtain

$$
U(\alpha, \varepsilon)<\frac{\varepsilon^{4}}{\sin \alpha}\left(\frac{141}{512}+\frac{7}{128} \alpha^{2}\right)-\frac{\varepsilon^{6}}{24 \sin \alpha}+\left[\varepsilon^{87}\right] .
$$

For $\alpha<0.156, \alpha / \sin \alpha$ is increasing, and so

$$
\frac{1}{\sin \alpha}<\frac{0.156}{\alpha \sin 0.156}<\frac{1.004 \cdots}{\alpha} .
$$

Therefore we obtain

$$
U(\alpha, \varepsilon)<\frac{28}{100 \alpha} \varepsilon^{4}-\frac{1}{24 \alpha} \varepsilon^{6}+\left[\varepsilon^{8}\right],
$$

and hence by numerical calculation we get

$$
U(\alpha, \varepsilon)<\frac{28}{100 \alpha} \varepsilon^{4}-\frac{1}{24 \alpha} \varepsilon^{6} .
$$

Since $\alpha<|A Q|$ we see that

$$
P\left(q_{1} q_{2} q_{3}\right)<3 U(\alpha, \varepsilon),
$$

and hence we obtain

LEMMA 7.2. For $\alpha<0.156,2 P\left(q_{1} q_{2} q_{3}\right)$ is estimated by

$$
2 P\left(q_{1} q_{2} q_{3}\right)<\frac{168}{100 \alpha} \varepsilon^{4}-\frac{1}{4 \alpha} \varepsilon^{6} .
$$

\section{§ 8. Proof of Theorem B.}

Proposition 8.1. For $N \geqq 100, A(\varepsilon, N)>\pi-M_{0}^{\prime}(\varepsilon, N ; A)$ holds.

Proof. By $N \geqq 100$ we obtain $\alpha<0.156$ and $\varepsilon<0.136$. Applying estimates (3.5), (3.9) and (6.4) and (7.8) to (4.7), we obtain 


$$
\begin{gathered}
A(\varepsilon, N)-\pi+M_{0}^{\prime}(\varepsilon, N ; A)=A(\varepsilon, N)-2 N \cdot P(\varepsilon)+2 P(\varepsilon, N, \Omega)-2 P\left(q_{1} q_{2} q_{3}\right) \\
>\pi N\left(\frac{1}{4} \varepsilon^{4}-\frac{1}{12} \varepsilon^{6}\right)-\left(1-\frac{98 \alpha}{100}\right) 2 N\left(\frac{\pi}{8} \varepsilon^{4}-\frac{\pi}{60} \varepsilon^{6}\right)-\frac{168}{100 \alpha} \varepsilon^{4}+\frac{1}{4 \alpha} \varepsilon^{6} \\
=\frac{98}{100} \alpha N\left(\frac{\pi}{4} \varepsilon^{4}-\frac{\pi}{30} \varepsilon^{6}\right)-\frac{1}{20} \pi N \varepsilon^{6}-\frac{168}{100 \alpha} \varepsilon^{4}+\frac{1}{4 \alpha} \varepsilon^{6} .
\end{gathered}
$$

By (3.8) and (4.5) we obtain

$$
\begin{aligned}
A(\varepsilon, N)-\pi+M_{0}^{\prime}(\varepsilon, N ; A) & >\frac{98}{100} \cdot \frac{12}{5 \alpha} \cdot \frac{78}{100} \varepsilon^{4}-\frac{\pi}{20}\left(2+\frac{1}{5} \varepsilon^{2}\right) \varepsilon^{4}-\frac{168}{100 \alpha} \varepsilon^{4} \\
& >\frac{183}{100 \alpha} \varepsilon^{4}-\frac{32}{100} \varepsilon^{4}-\frac{168}{100 \alpha} \varepsilon^{4} \\
& =\frac{15-32 \alpha}{100 \alpha} \varepsilon^{4}>0
\end{aligned}
$$

Proof of Theorem B. By Proposition 8.1 we see that $M_{0}^{\prime}(\varepsilon, N ; A)>\pi-$ $A(\varepsilon, N)$ holds. Since $M_{0}^{\prime}(\varepsilon, N ; A)$ is the mean value, we have some plane $L$ through $O$ and $A$ such that

$$
\operatorname{Area}\left(K\left(\varepsilon, N, \Theta_{0}^{\prime}\right) \cap L\right)>\pi-A(\varepsilon, N) \text {. }
$$

Let $\Theta$ be an $\varepsilon$-proper distribution of $2 N$ points on $S^{2}(1)$, and let $q$ be a point of $S^{2}(1)$. By $M(q)=M(\varepsilon, N, \Theta ; q)$ we denote the rotational mean value at $q$, i.e., the mean value of $\operatorname{Area}\left(K(\varepsilon, N, \Theta) \cap L_{q}\right)$ with respect to planes $\left\{L_{q}\right\}$ which contain the line $q O$. If we consider $M(q)$ as a function on $S^{2}(1), \pi-M(q)$ takes big value at $q$ if relatively many points of $\Theta$ are distributed near $q$, or if $q$ is very near some $p_{k}$ of $\Theta$. Theorem B implies that even if $\Theta$ is nearly homogeneous, the variation of $M(q)$ with respect to $q$ is not so small.

Observations for the case where $N$ is small and Theorem B lead us to the following conjecture.

CONJECTURE. For an e-proper distribution $\Theta$ of $2 N$ points on $S^{2}(1)$. $\operatorname{Area}(K(\varepsilon, N, \Theta) \cap L)<\operatorname{Area}\left(B^{3}(R) \cap L\right)$ for each $L$ may imply

$$
\operatorname{Vol}(K(\varepsilon, N, \Theta))<\operatorname{Vol}\left(B^{3}(R)\right) \text {. }
$$

As a remark we prove the following.

Proposition 8.2. Let $\varepsilon$ and $N$ be given so that $2 N$ points can be $\varepsilon$-properly distributed on $S^{2}(1)$. Then;

(i) There exists an $\varepsilon$-proper distribution $\Theta_{*}$ of $2 N$ points on $S^{2}(1)$ such that the maximum value of the rotational mean value function $M_{*}(q)$ is not greater than the maximum value of $M(q)$ for any other $\varepsilon$-proper distribution $\Theta$ of $2 N$ points on $S^{2}(1)$.

(ii) There exists an $\varepsilon$-proper distribution $\Theta^{*}$ of $2 N$ points on $S^{2}(1)$ such that 
the maximum value of $\operatorname{Area}\left(K\left(\varepsilon, N, \Theta^{*}\right) \cap L\right)$ with respect to $\{L\}=R P^{2}$ is not greater than the maximum value of $\operatorname{Area}(K(\varepsilon, N, \Theta) \cap L)$ with respect to $\{L\}$ for any other $\varepsilon$-proper distribution $\Theta$ of $2 N$ points on $S^{2}(1)$.

Proof. Let $\Psi$ be a subset of $S^{2}(1) \times S^{2}(1) \times \cdots \times S^{2}(1)(N$ times) composed of elements $\left(p_{1}, p_{2}, \cdots, p_{N}\right)$ such that $\left|p_{k}\left( \pm p_{l}\right)\right| \geqq 2 \varepsilon$ for $1 \leqq k<l \leqq N$. Then $\Psi$ is compact. The rotational mean value function $M(\varepsilon, N, \Theta ; q)$ is a continuous function on $\Psi \times S^{2}(1)$. We define $\Lambda(\varepsilon, N, \Theta)$ by

$$
\Lambda(\varepsilon, N, \Theta)=\max _{q \in S^{2}(1)}\{M(\varepsilon, N, \Theta ; q)\} .
$$

Then $\Lambda(\varepsilon, N, \Theta)$ is a continuous function on $\Psi$. Therefore we have some $\Theta_{*} \in \Psi$, which attains the minimum of $\Lambda(\varepsilon, N, \Theta)$. This proves (i).

(ii) is easily proved.

\section{§9. Appendix.}

It is clear that if $N$ is not so large and $\varepsilon$ is very small then for any $\varepsilon$ proper distribution of $2 N$ points on $S^{2}(1)$ we can find some $L$ such that $K(\varepsilon, N, \Theta) \cap L=B^{3}(1) \cap L$.

Let $\Theta$ be an $\varepsilon$-proper distribution of $2 N$ points on $S^{2}(1)$. Even if $\operatorname{Area}(K(\varepsilon, N, \Theta) \cap L)<\pi$ holds for any $L$, we see that the variation of $\operatorname{Area}(K(\varepsilon, N, \Theta) \cap L)$ is big, if $N$ is not so large. To show this we give two examples corresponding to the closest packings of equal circles on $S^{2}(1)$.

$\langle 9-1\rangle$ Octahedron.

Consider an octahedron inscribed in $S^{2}(1)$. Vertices define an $\varepsilon$-proper distribution of six points on $S^{2}(1)$ with $\varepsilon=\pi / 4$. Let $\Theta=\{ \pm X, \pm Y, \pm A\}$ where $X=(1,0,0), Y=(0,1,0)$ and $A=(0,0,1)$. Let $L_{0}$ be the plane passing through $Y,-Y$ and $(\sqrt{2} / 2,0, \sqrt{2} / 2)$. Then

$$
\begin{aligned}
\operatorname{Area}\left(K(\pi / 4,3, \Theta) \cap L_{0}\right) & =2.5707 \cdots, \\
\pi R(\pi / 4,3)^{2} & =2.3613 \cdots .
\end{aligned}
$$

Therefore $\operatorname{Area}(K(\pi / 4,3, \Theta) \cap L)<\pi R(\pi / 4,3)^{2}$ does not hold for $L_{0}$.

Notice that $\Theta$ corresponds to the closest packing of equal six circles on $S^{2}(1)$. As for closest packing, see for example [5] and [6] or references there.

$\langle 9-2\rangle$ Icosahedron.

Consider an icosahedron inscribed in $S^{2}(1)$. Vertices define an $\varepsilon$-proper distribution of $\Theta$ of eleven points on $S^{2}(1)$ with $\varepsilon=0.5535 \cdots$. Let $p_{1} \in \Theta$ and let $L_{0}$ be the plane orthogonal to $p_{1}\left(-p_{1}\right)$. Then

$$
\begin{aligned}
\operatorname{Area}\left(K(\varepsilon, 6, \Theta) \cap L_{0}\right) & =2.9389 \cdots, \\
\pi R(\varepsilon, 6)^{2} & =2.7281 \cdots .
\end{aligned}
$$

Therefore $\operatorname{Area}(K(\varepsilon, 6, \Theta) \cap L)<\pi R(\varepsilon, 6)^{2}$ does not hold for $L_{0}$. 
$\Theta$ corresponds to the closest packing of equal twelve circles on $S^{2}(1)$. $\langle 9-3\rangle$ Non-symmetric closest packing.

Let $p_{1}=(\sin b, 0, \cos b)$ with $\sin ^{2} b=(8-2 \sqrt{2}) / 7$. By $\pi / 2-, \pi-$, and $3 \pi / 2-$ rotation of $p_{1}$ around the $x^{3}$-axis, we define $p_{2}, p_{3}$, and $p_{4}$. By $\pi / 4$-rotation of $-p_{1},-p_{2},-p_{3}$, and $-p_{4}$ around the $x^{3}$-axis, we define $q_{1}, q_{2}, q_{3}$, and $q_{4}$. Then $\Sigma=\left\{p_{1}, p_{2}, p_{3}, p_{4}, q_{1}, q_{2}, q_{3}, q_{4}\right\}$ defines the closest packing of equal eight circles on $S^{2}(1)$ with $\varepsilon$ such that $\cos ^{2} \varepsilon=(3+\sqrt{2}) / 7$, i. e., $\varepsilon=0.6532 \cdots . \quad \Sigma$ is not centrally symmetric. By $K(\varepsilon, \Sigma)$ we denote the convex body obtained from $B^{3}(1)$ by removing eight spherical caps of $B^{3}(1)$ of angular radius $\varepsilon$ corresponding to $\Sigma$. Let $L_{0}$ be the plane passing through $A, q_{1}$, and $q_{3}$. Then

$$
\begin{array}{r}
\operatorname{Area}\left(K(\varepsilon, \Sigma) \cap L_{0}\right)=2.8003 \cdots, \\
\pi R(\varepsilon, 4)^{2}=2.6234 \cdots .
\end{array}
$$

\section{REFERENCES}

[1] A.L. BEsse, Manifolds all of whose geodesics are closed, Ergeb. Math., no. 93, Springer, 1978

[2] T. Bonnesen and W. Fenchel, Theorie der konvexen Körper, Berlin, 1934.

[3] H. Busemann, Volumes in terms of concurrent cross-sections, Pacific J. Math., 3 (1953), 1-12.

[4] H. Busemann and C. M. Petty, Problems on convex bodies, Math. Scand., 4 (1956), 88-94.

[5] B.W. Clare and D.L. Kepert, The closest packing of equal circles on sphere, Proc. R. Soc. London, A405 (1986), 329-344.

[6] L. DANZER, Finite point-sets on $S^{2}$ with minimum distance as large as possible, Discrete Math., 60 (1986), 3-66.

[7] P. Funk, Uber eine geometrische Anwendung der Abelschen Integralgleichung, Math. Ann., 77 (1916), 129-135.

[8] D. G. LARMAN, Recent results in convexity, Proc. Intl. Congress Math., Helsinki, Vol. 1 (1978), 429-434.

[9] D. G. LARMAN AND C.A. Rogers, The existence of a centrally symmetric convex body with central sections that are unexpectedly small, Mathematika, 22 (1975), 164-175.

Department of Mathematics

TOKYo Institute of TECHNOLOGY

TOKYO, JAPAN 